\title{
The efficacy of ma-huang-tang (maoto) against influenza
}

\author{
Mizue Saita, Toshio Naito, Soushin Boku, Yukiko Watanabe, Mai Suzuki, Fukuko Oka, \\ Miki Takahashi, Takako Sakurai, Eiichirou Sugihara, Tomomi Haniu, Yuki Uehara, \\ Kazunori Mitsuhashi, Hiroshi Fukuda, Hiroshi Isonuma, Keiko Lee, Hiroyuki Kobayashi
}

Department of General Medicine, Juntendo University School of Medicine, Tokyo, Japan;

*Corresponding Author: koba@med.juntendo.ac.jp

Received 1 February 2011; revised 20 April 2011; accepted 27 April 2011.

\section{ABSTRACT}

In this study, we compared Ma Huang Tang (maoto), a traditional Japanese medicine (Kampo), with antiviral drugs to evaluate their respective and combined effect on the duration of fever and other subjective symptoms of influenza. Forty-five patients enrolled in this randomized control trial had positive type A influenza on rapid influenza antigen test, provided written consent, and sought treatment at Juntendo University Hospital between November 2008 and March 2009. Using a computer-gen-erated list, patients were randomly assigned to one of the four intervention groups: 1): maoto (TJ-27), 9 subjects; 2): Tamiflu (oseltamivir), 13 subjects; 3): Relenza (zanamivir), 6 subjects; and 4): maotoloseltamivir combination, 9 subjects. Six outcome measures were evaluated, including fever, myalgia, headache, arthralgia, fatigue, and cough. Statistical differences were determined by the Bonferroni-adjusted t-test for multiple comparisons. Our results showed that there were no significant differences among the four groups in the time-course profile of fever and the number of days until fever resolution since treatment was initiated. In addition, no significant intergroup differences were detected in the number of days until resolution of myalgia, headache, fatigue, and cough. However, the maoto group reported a more rapid improvement in joint pain than the oseltamivir group $(P=$ 0.01). In conclusion, maoto showed comparable efficacy as antiviral medications in reducing fever and influenza symptoms. As serious concerns over the indiscriminate use, adverse reactions, and resistance to current antiviral drugs continue to grow, maoto may serve as an elegant option for the treatment of influenza.
Keywords: Ma Huang Tang (Maoto); Influenza; Joint Pain; Oseltamivir and Zanamivir

\section{INTRODUCTION}

The influenza virus was first isolated by Smith, Andrews, and Laidlow in 1933. Six decades have passed and influenza continues to circulate worldwide despite substantial research and advances in early and effective vaccination use [1]. In the wake of WHO Declaration of "Pandemic Phase 6 for Novel Influenza A (H1N1)", an expedited search for potent antiviral agents was summoned [2]. Antiviral drugs remain the mainstay of influenza treatment but their use has given rise to a number of problems. The widespread and often indiscriminate use of antiviral drugs has generated reports on accelerated drug resistance in viruses [3-5] and adverse drug reactions notwithstanding unclear causal relationship [6,7].

Human influenza infection is predominantly an upper respiratory tract infection, yet most patients present and complain of various systemic symptoms. Despite empirically demonstrated efficacy of Kampo medicines in relieving systemic manifestations, such data often fall short as clinical recommendations. Of the two traditional Japanese (Kampo) medicines-maoto and chikujyountanto-which are covered under the National Health Insurance scheme for the treatment of influenza, we sought to investigate the efficacy of maoto as the front-line Kampo drug prescribed during the acute stage of influenza.

During the 2008-2009 influenza season, we prospectively compared the efficacy of maoto with antiviral drugs in reducing fever and flu symptoms (myalgia, headache, arthralgia, fatigue, and cough) in adult patients with seasonal influenza type A.

The Juntendo University School of Medicine Hospital Institutional Review Board reviewed and approved all study procedures (approval number: 208-037). 


\section{MATERIALS AND METHODS}

Adult patients ages 20 and over with a positive nasopharyngeal swab for influenza type A on rapid diagnostic test (rapid flu test, Sekisui Medical Co., Ltd) and sought treatment at the Department of General Medicine at Juntendo University Hospital between November 2008 and March 2009 enrolled in the study. A total of 45 patients provided written consent (mean age $32.4 \pm 11.5$ years: 21 men, 24 women) upon full comprehension of the study procedures. A computer-generated list was used to assign patients to four different interventions (I: maoto (TJ-27), II: Tamiflu (oseltamivir), III: Relenza (zanamivir), and IV: maoto/oseltamivir combination therapy), and the efficacy of each treatment was evaluated as a randomized control study. Main outcome measures included fever onset, arthralgia, myalgia, headache, cough, and fatigue. Patients were instructed to keep a record of their symptoms on an evaluation postcard and to rate the intensity of their arthralgia, myalgia, headache, cough, and fatigue on a 5-point scale (score $0-4)$. They were also advised to track their fever, medication schedule, and body temperature for five days after their diagnosis. The time it took for the highest score to drop to zero for a given symptom was defined as the "number of days until symptom relief"; the symptom severity was calculated as the difference between the highest and lowest scores and termed "reduction in symptom score". The product of the "number of days until symptom relief" and "reduction in symptom score" was statistically analyzed using the Bonferroni-adjusted t-test for multiple comparisons.

Eight out of 45 patients failed to return their evaluation postcards (I: 2 subjects, II: 1 subject, III: 3 subjects, IV: 2 subjects), resulting in a final analysis based on 37 patients (I: 9 subjects, II: 13 subjects, III: 6 subjects, and IV: 9 subjects).

\section{RESULTS AND DISCUSSION}

At the time of treatment allocation, no significant differences were observed in age, onset and duration of fever, and vaccination rate among the patients (Table 1).
Maoto-treated subjects (I and IV) had near-equivalent reduction in fever (body temperature: fever duration) relative to those of oseltamivir-(III) and zanamivir-(IV) treated subjects, thus no significant differences in antipyretic effects were found among the three groups (Figure 1) $(p=0.90)$. Further, all groups showed comparable effects in alleviating headache, cough, fatigue, and myalgia (Figure 2), with an exception of a more rapid improvement in joint pain in the maoto group than the oseltamivir group (Figure 3) $(\mathrm{P}=0.02)$. However, without a placebo group to compare against, we were unable to determine whether maoto could alleviate joint pain better than placebo. Nevertheless, Ito et al. previously discovered that the duration of joint pain in the placebo group without treatment was 2.8 days $( \pm 1.7 \mathrm{SD})$ whereas it was 3.5 days $( \pm 2.0)$ in the amantadine group in a RCT that evaluated the efficacy of amantadine against influenza type A. Thus, a joint pain of 2 day $( \pm 1.1)$ duration in the maoto group in our study appears to be shorter than the duration observed in the placebo group in Ito et al.'s study [8].

With the H1N1 influenza outbreak, the shortage of antiviral drugs has become a burgeoning public health

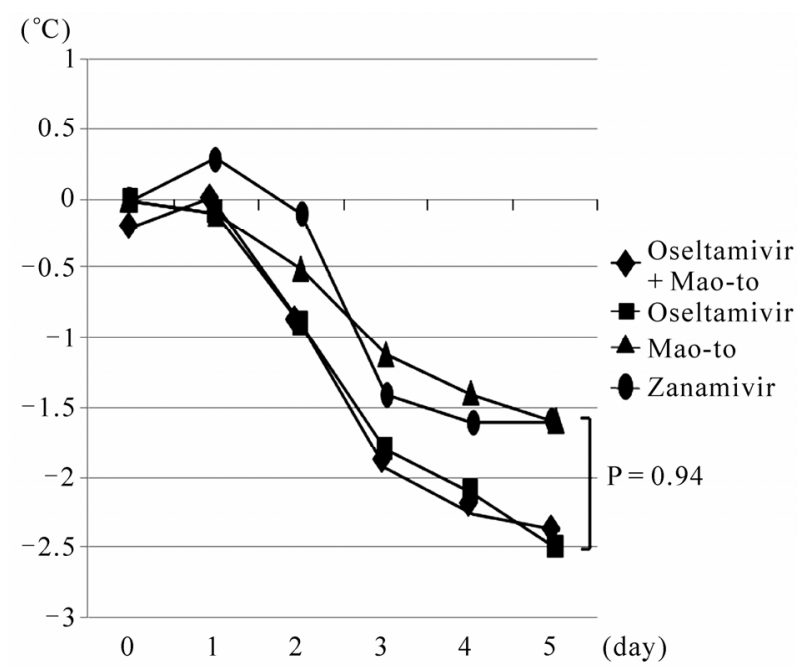

Figure 1. Time-course profiles of fever of the four treatment groups.

Table 1. Demographics and medical history.

\begin{tabular}{lcccc}
\hline & I Mao-to & II Oseltamivir & III Zanamivir & IV Oseltamivir and Mao-to \\
\hline Number & $\mathrm{n}=9$ & $\mathrm{n}=13$ & $\mathrm{n}=6$ & $\mathrm{n}=9$ \\
Age $\left(\right.$ Mean age $\pm \mathrm{SD}^{*}$ ) & $31.2 \pm 10.4$ & $33.3 \pm 14.8$ & $34.3 \pm 6.07$ & $31.5 \pm 8.5$ \\
Sex (n) & Male: 2 & Male: 7 & Male: 3 & Male: 4 \\
Mean (SD) Duration of Fever before Medication & Female: 7 & Female: 6 & Female: 3 & Female: 5 \\
Mean (SD) Baseline Temperature $\left({ }^{\circ} \mathrm{C} \pm \mathrm{SD}^{*}\right)$ & $14.25 \pm 7.63$ & $25.8 \pm 15.59$ & $21.0 \pm 11.91$ & $24.0 \pm 14.36$ \\
Number of Patients Immunized with Current Influenza Vaccine & $38.0 \pm 0.66$ & $38.7 \pm 0.60$ & $38.0 \pm 0.61$ & $38.4 \pm 0.66$ \\
\hline
\end{tabular}



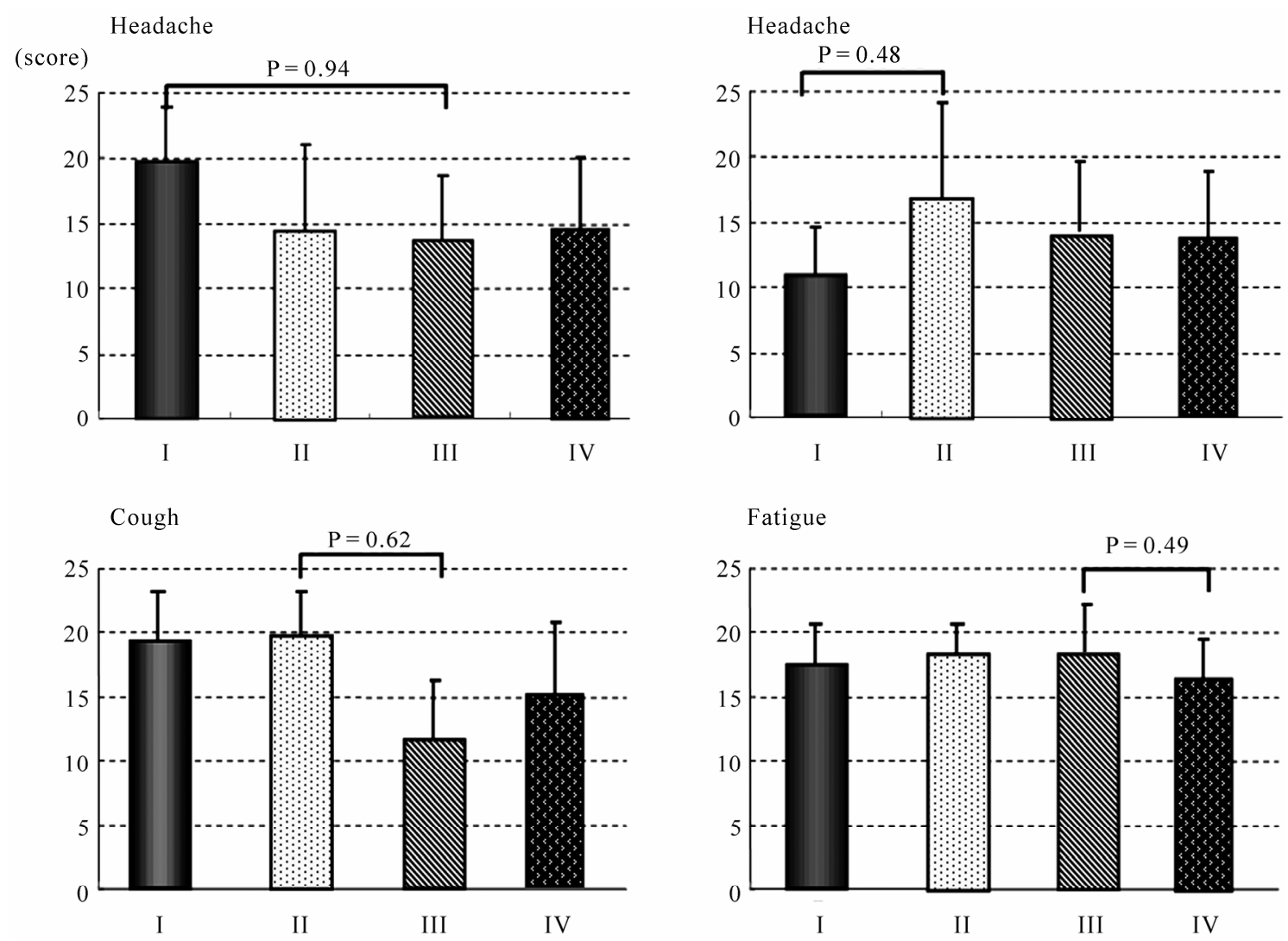

Figure 2. Severity of headache, muscle pain, cough and fatigue.



Figure 3. Severity of joint pain.

problem. Moreover, unequal dispensation and inadequate access to antiviral drugs have been speculated as causing an aggravation of the disease in compromised patients.

Much controversy exists over oseltamivir, the first-line agent for influenza, regarding its dosage, development of drug-resistant strains, and adverse drug reactions. According to published data, Japan comprises $77 \%$ of all oseltamivir usage in the world, while the United States occupies the $22 \%$ of world consumption. Between 2008 and 2009, the majority of H1N1 viruses isolated worldwide were reportedly oseltamivir-resistant strains; in Japan, the resistance rate reached 99.6\% (1234/1239) by March 2009 [9].

In contrast, maoto, which is composed of mao (Ephedrae herba), annin (Armeniacae semen), keishi (Cinnamomi cortex), and kanzo (Glycyrrhizae radix), has been long touted as the leading prescription for early stages of common cold in the ancient Chinese medical literature Shang Han Lun. Influenza primarily affects the upper respiratory tract, yet patients often present and suffer from systemic symptoms. Kampo medicines have putative ability to address generalized symptoms through the synergy of multiple botanical raw materials. For instance, a preliminary investigation of antiviral properties of Kampo medicines showed that keishi exerted a dose-dependent antiviral effect [10]. Hattori et al. further suggested that the antiviral effect of keishi against multiple strains were associated with its suppressive effect on various aspects of endocytosis [11].

In the present study, maoto showed comparable efficacy as antiviral drugs in influencing the duration of fever and subjective symptoms, including headache, cough, fatigue, and myalgia. Also, maoto demonstrated 
greater efficacy than oseltamivir or zanamivir in improving joint pain. This finding is corroborated by Kubo and Nishimura who found that treatment with maoto or maoto-oseltamivir combination was more effective than oseltamivir alone in shortening the duration of fever [12]. If we take into account the fact that maoto has been historically used to treat cold symptoms based on (symptom) pattern diagnosis in Kampo medicine, it is not surprising to discover its efficacy to be on par with antivirals which are specifically designed to suppress viral replication. Hence, our results are clinically significant, given the scarcity of data on maoto's efficacy in influenze from prospective randomized trials.

Oseltamivir showed strong but statistically insignificant antipyretic effect. Figure 1 depicts the antipyretic effect of both oseltamivir administered groups (II and IV).

Zanamivir-treated group showed a rapid improvement in cough than the other three groups. Though statistically unremarkable, our results undergird the traditional use of Zanamivir as a potent respiratory agent.

While efforts to discover novel antiviral agents and oseltamivir-related problems continue to attract attention, maoto's effect in influenza as endorsed by the NHI and observed in our prospective study are useful information for both healthcare practitioners and patients.

Except for one patient developing nausea from the maoto's distinct odor, no other serious adverse events were reported during the study. Based on our observations, nine patients on maoto/oseltamivir combination therapy, who were anticipated to achieve a greater overall symptom relief than patients in other treatment groups, did not demonstrate apparent benefits of the combination therapy.

The absence of a control group with inert placebo and a control group without treatment due to insurance purposes were two important caveats in this study. Additionally, we did not investigate the effect of maoto in other influenza types because all patients recruited for the study were uniformly infected with type A virus, although Toriumi et al. had previously found that maoto was as effective as oseltamivir and zanamivir in reducing fever in pediatric patients with type B influenza [13]. As the speed and routes of $\mathrm{H} 1 \mathrm{~N} 1$ propagation gain momentum, clinically-oriented studies based on isolated strains may halt further global catastrophe.

In summary, we found that maoto improved systemic influenza symptoms during the active disease phase as effectively, if not, better than antiviral drugs. Moreover, because none of the patients receiving maoto or maoto/ antiviral combination therapy suffered adverse drug reactions, maoto appeared to have a slightly wider safety margin than antiviral drugs. Maoto may curb the devel- opment of drug resistance to antivirals caused by their misuse and overuse, and serve as an elegant option for the treatment of influenza.

\section{REFERENCES}

[1] WHO (2008) Brief summary of analyses of the 2007 survey on the capacity of National Influenza Centres. World Health Organization Geneva, 83, 133-1448.

[2] WHO (2009) Interim planning considerations for mass gatherings in the context of pandemic (H1N1) 2009 influenza.

[3] Dharan, N.J., Gubareva, L.V., Meyer, J.J., OkomoAdhiambo, M., McClinton, R.C., Marshall, S.A., St. George, K., Epperson, S., Brammer, L., Klimov, A.I., Bresee, J.S., Fry, A.M. (2009) Infections with oseltamivir-resistant influenza $\mathrm{A}(\mathrm{H} 1 \mathrm{~N} 1)$ virus in the United States. Journal of the American Medical Association, 301, 1034-1041. doi:10.1001/jama.2009.294

[4] Hatoyama, S. and Koeike, K. (2009) Development and management of oseltamivir-resistant influenza. The Japanese Society of Internal Medicine, 98, 1733-1741.

[5] Centers for Disease Control and Prevention (CDC) (2006) High levels of adamantane resistance among influenza A (H3N2) viruses and interim guidelines for use of antiviral agents-United States, 2005-2006 influenza season. Morbidity and Mortality Weekly Report, 55, 44-46.

[6] IDSC (2008) Infectious Disease Surveillance Center, 2007/2008 influenza season, Japan. Infectious Agents Surveillance Report, 29, 297-298.

[7] World Health Organization 2009) Influenza A(H1N1) virus resistance to oseltamivir-2008/2009 influenza season, northern hemisphere.

[8] Ito, S., Iijima, N., Kanemaki, K., Hayashi, S., Hujii, S., Watanabe, T., Hosokawa, T. and Kakehi, K. (2000) Therapeutic efficacy of amantadine hydrochloride in patients with epidemic influenza A virus infection. Nihon Kokyuki Gakkai Zasshi, 38, 897-902.

[9] Chiharu, K., Masatsugu, O., Miwako, S., Yuzo, N., Makoto, U., Takako, O., Mami, I., Takahiro, T. and Masato, T. (2009) Isolation of oseltamivir-resistant influenza A/H1N1 virus of different origins in Yokohama city, Japan, during the 2007-2008 influenza season. Japanese Journal of Infectious Diseases, 62, 83-86.

[10] Hayashi, K., Imanishi, N., Kashiwayama, Y., Kawano, A., Terasawa, K., Shimada, Y. and Ochiai, H. (2007) Inhibitory effect of cinnamaldehyde, derived from Cinnamomi cortex, on the growth of influenza $\mathrm{A} / \mathrm{PR} / 8$ virus in vitro and in vivo. Antiviral Research, 74, 1-8. doi:10.1016/j.antiviral.2007.01.003

[11] Hattori, T. (2007) Hochuekito for biodefense and viral infections. Mebio, 24, 16-21.

[12] Kubo, T. and Nishimura, H. (2007) Antipyretic effect of Mao-to, a Japanese herbal medicine, for treatment of type A influenza infection in children. Phytomedicine, 14, 96-101 (article in Japanese with English abstract). doi:10.1016/j.phymed.2006.09.015

[13] Toriumi, Y., Takahashi, I., Kamei, T. and Murata, K. (2010) Antipyretic effect of Maoto in treatment of type B influenza infection in children. Journal of Epidemiology, 20, 360, (Article and abstract in Japanese). 